\title{
Periodic points for amenable group actions on uniquely arcwise connected continua
}

\author{
ENHUI SHI $\dagger$ and XIANGDONG YE $\ddagger$ \\ $\dagger$ School of Mathematical Sciences, Soochow University, Suzhou 215006, P. R. China \\ (e-mail: ehshi@suda.edu.cn) \\ $\$ W u$ Wen-Tsun Key Laboratory of Mathematics, USTC, Chinese Academy of Sciences \\ and Department of Mathematics, University of Science and Technology of China, Hefei, \\ Anhui 230026, P. R. China \\ (e-mail: yexd@ustc.edu.cn)
}

(Received 4 June 2020 and accepted in revised form 17 June 2020)

Abstract. We show that any action of a countable amenable group on a uniquely arcwise connected continuum has a periodic point of order $\leq 2$.

Key words: amenable group, periodic point, dendrite, continuum 2020 Mathematics Subject Classification: 37B05 (Primary); 54F50 (Secondary)

\section{Introduction}

In this section we will give some basic notions we shall use in the paper. Moreover, we will state the background and the main theorem.

1.1. Basic notions. Let $X$ be a topological space and let $G$ be a group. A homomorphism $\phi$ of $G$ into the semigroup of all continuous self-mappings of $X$ is called an action of $G$ on $X$. (Note that each $\phi(g)$ is a homeomorphism of $X$ with a continuous inverse $\phi\left(g^{-1}\right)$.) For brevity, we usually use $g x$ or $g(x)$ instead of $\phi(g)(x)$ for $g \in G$ and $x \in X$. The orbit of $x \in X$ under the action of $G$ is the set $G x \equiv\{g x: g \in G\}$. If $G x$ is finite, then $x$ is called a periodic point of $\phi$ and the cardinality $n$ of $G x$ is called the order of $x$; we also say that $x$ is an n-periodic point (respectively a fixed point if $n=1$, i.e. if $g x=x$ for all $g \in G)$. A subset $Y$ of $X$ is called $G$-invariant if $g(Y) \subset Y$ for all $g \in G$. A Borel measure $\mu$ on $X$ is called $G$-invariant if $\mu(g(A))=\mu(A)$ for every Borel set $A$ in $X$ and every $g \in G$.

Amenability was first introduced by von Neumann. Recall that a group $G$ is called an amenable group if there is a sequence of finite sets $F_{i}(i=1,2,3, \ldots)$ with $\bigcup_{i=1}^{\infty} F_{i}=$ $G$ such that $\lim _{i \rightarrow \infty}\left(\left|g F_{i} \triangle F_{i}\right| /\left|F_{i}\right|\right)=0$ for every $g \in G$, where $\left|F_{i}\right|$ is the number of 


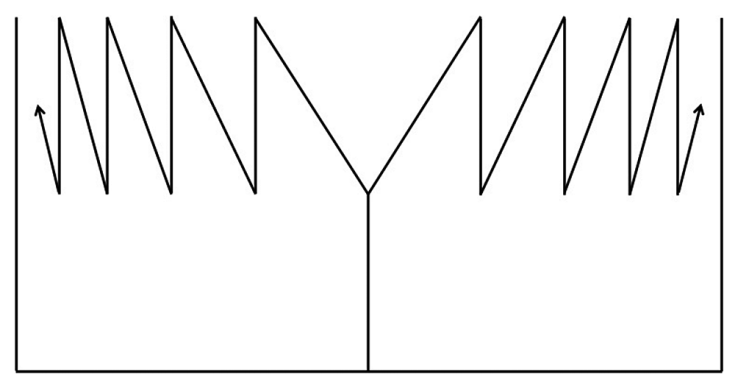

FIGURE 1. A uniquely arcwise connected continuum which is the union of two copies of the Warsaw circle.

elements in $F_{i}$; the set $F_{i}$ is called a Følner set. It is well known that solvable groups and finite groups are amenable; every subgroup of an amenable group is amenable. It is also known that any group containing a free non-commutative subgroup is not amenable. An important characterization of a countable amenable group is that $G$ is amenable if and only if every action of $G$ on a compact metric space $X$ has a $G$-invariant Borel probability measure on $X$. One may consult [8] for a systematic introduction to amenability.

By a continuum, we mean a connected compact metric space. A continuum is non-degenerate if it is not a single point. An arc is a continuum which is homeomorphic to the closed interval $[0,1]$. A continuum $X$ is uniquely arcwise connected if for any two points $x \neq y \in X$ there is a unique arc $[x, y]$ in $X$ which connects $x$ and $y$. A dendrite is a locally connected, uniquely arcwise connected continuum. A tree is a dendrite which is the union of finitely many arcs. Clearly, the class of uniquely arcwise connected continua is strictly larger than that of dendrites. For example, the Warsaw circle is uniquely arcwise connected but not locally connected.

We provide in the following an example of a uniquely arcwise connected continuum contained in the plane, which will be repeatedly mentioned throughout the paper.

Example 1.1. (See Figure 1.) Let $\mathbb{R}^{2}$ be the Euclidean plane. For each positive integer $n$, let $I_{n}, I_{-n}, J_{n}$, and $J_{-n}$ be the segments between $((n-1) / n, 0)$ and $(n /(n+1), 1)$, between $(-(n-1) / n, 0)$ and $(-n /(n+1), 1)$, between $(n /(n+1), 0)$ and $(n /(n+1), 1)$, and between $(-n /(n+1), 0)$ and $(-n /(n+1), 1)$, respectively. Let $S^{-}=\bigcup_{n=1}^{\infty}\left(I_{-n} \cup J_{-n}\right)$ and $S^{+}=\bigcup_{n=1}^{\infty}\left(I_{n} \cup J_{n}\right)$. Let $L, B, R$, and $M$ be the segments between $(-1,-1)$ and $(-1,1)$, between $(-1,-1)$ and $(1,-1)$, between $(1,-1)$ and $(1,1)$, and between $(0,-1)$ and $(0,0)$, respectively. Let $X=L \cup B \cup R \cup M \cup S^{-} \cup S^{+}$. Then $X$ is a uniquely arcwise connected continuum which is not locally connected.

1.2. Background and the main theorem. For an action of a group $G$ on a topological space $X$, an interesting question is whether there exists a fixed point or a periodic point of the action. The answer to this question certainly depends on the topology of $X$ and involves the algebraic structure of $G$.

In 1975, Mohler proved in [6] that every homeomorphism (i.e. $\mathbb{Z}$-action) on a uniquely arcwise connected continuum has a fixed point, which answered a question proposed by Bing (see [1]). In 2009, this result was generalized to nilpotent group actions by Shi and 
Sun (see [9]). In 2010, Shi and Zhou further showed that every solvable group action on such continua has a periodic point of order $\leq 2$ (see [11]). In 2017, Shi and Ye proved that every countable amenable group action on a dendrite has a periodic point of order $\leq 2$ (see [10]). One may consult $[2,4,5]$ for some interesting discussions about fixed point theory for mappings on uniquely arcwise connected continua. We also remark that a continuous map on a uniquely arcwise connected continuum may have no fixed points (see [12]).

We get the following theorem in this paper, which generalizes all the corresponding results stated above. (Noting that the integer group is amenable, we see by the following theorem that any integer group action on a uniquely arcwise connected continuum preserves either a point or an arc, which implies the existence of fixed points.)

THEOREM 1.2. Any action of a countable amenable group on a uniquely arcwise connected continuum has a periodic point of order $\leq 2$.

We should note that the set of end points and the set of branch points of a uniquely arcwise connected continuum $X$ are each homeomorphism invariants. So, if the set of end points of $X$ is finite, or if the set of branch points of $X$ is finite but non-empty, then any group action on $X$ admits periodic points, which easily implies the existence of a periodic point of order $\leq 2$. However, a uniquely arcwise connected continuum may have infinitely many branch points and even uncountably many end points. This complicates the problem. We also remark that if the acting group $G$ is the free group $\mathbb{Z} * \mathbb{Z}$ (not amenable), then there does exist counterexamples of $G$-actions on some dendrites without periodic points (see [10, Theorem 6.1]); if $G$ is the solvable group $(\mathbb{Z} / 2 \mathbb{Z}) \ltimes \mathbb{Z}$, then $G$ can act on the closed interval $[0,1]$ with a periodic point of order two and with no fixed points (see [11, Remark 1.3]).

The proofs of the main theorems in $[9,11]$ heavily rely on the existence of a decreasing sequence of commutator subgroups ending at the identity, which reduces the proof to the case of abelian group actions; however, such subgroup sequences do not exist in amenable groups in general. So, we have to develop some new techniques to overcome this difficulty.

In $\S 2$, we introduce some notions and results concerning the structure and mapping properties of uniquely arcwise connected continua. In $\S 3$, we construct a convex metric on a special class of arcwise connected subsets of uniquely arcwise connected continua and study their completions with respect to this metric. Specially, we establish a connection between the group actions on dendrites and those on uniquely arcwise connected continua. Based on the connection established in $\S 3$ and the main theorem in [10], we prove Theorem 1.2 in $\S 4$.

\section{Preliminaries}

In this section we will introduce several notions which are important for the study of our question, including the convex hulls, dendrites, rays and lines in a compact metric space, and the quasi-retractions.

2.1. Convex hulls. Let $X$ be a uniquely arcwise connected continuum. If $S$ is a subset of $X$, we denote by $[S]$ the intersection of all arcwise connected subsets containing $S$ and call 
it the convex hull of $S$ in $X$. Clearly, $[S]$ is the minimal one among all the arcwise connected subsets which contain $S$. We remark here that $[S]$ need not be compact in general. Denote by $[a, b]$ the unique arc in $X$ connecting $a$ and $b$ and by $[a, b),(a, b]$, and $(a, b)$ the sets $[a, b]-\{b\},[a, b]-\{a\}$, and $[a, b]-\{a, b\}$, respectively.

The following lemma is clear.

LEMMA 2.1. If $S$ is a finite set in a uniquely arcwise connected continuum $X$, then $[S]$ is a tree.

Example 2.2. In Example 1.1, $\left[S^{-}\right]=S^{-}$is not compact; if $S$ is the finite set consisting of points $(-1,1),(1,1)$, and $(0,0)$, then $[S]=L \cup B \cup R \cup M$, which is a tree.

2.2. Dendrites. Let $X$ be a dendrite and let $x \in X$. We use $\operatorname{ord}(x, X)$ to denote the cardinality of the set of all components of $X-\{x\}$, which is called the order of $x$ in $X$. The point $x$ is a cut point if $\operatorname{ord}(x, X)=2$; is a branch point if $\operatorname{ord}(x, X) \geq 3$; and is an end point if $\operatorname{ord}(x, X)=1$. For a non-degenerate dendrite $X$, there are at most countably many branch points, there are uncountably many cut points, and there always exist end points. One may consult [7] for more properties of dendrites.

Proposition 2.3. Let $f$ be a homeomorphism on a non-degenerate dendrite X. Suppose that $e$ is an end point of $X$ such that $f(e)=e$. Then there is $u \in X, u \neq e$ such that either $f([e, u]) \subset[e, u]$ or $f^{-1}([e, u]) \subset[e, u]$. Moreover, there is $u^{\prime} \in(e, u]$ such that $\left[e, u^{\prime}\right] \cup$ $f\left(\left[e, u^{\prime}\right]\right) \cup f^{-1}\left(\left[e, u^{\prime}\right]\right) \subset[e, u]$.

Proof. Fix a point $v \neq e \in X$. Since $e$ is an end point and $f(e)=e$, there is $w \neq e$ such that $[e, w]=[e, v] \cap[e, f(v)]$. Let $u=f^{-1}(w)$. If $[e, u] \subset[e, w]$, then $f^{-1}([e, u]) \subset$ $f^{-1}([e, w])=[e, u]$; if $[e, w] \subset[e, u]$, then $f([e, u])=[e, w] \subset[e, u]$.

Moreover, if $f([e, u]) \subset[e, u]$, we choose $u^{\prime} \in(e, u]$ with $f^{-1}\left(u^{\prime}\right)=u$ and, if $f^{-1}([e, u]) \subset[e, u]$, we choose $u^{\prime} \in(e, u]$ such that $f\left(u^{\prime}\right)=u$. Then $u^{\prime}$ is the point we want.

The following two corollaries follow immediately from Proposition 2.3.

COROLlary 2.4. Let $f_{1}, \ldots, f_{n}$ be homeomorphisms on a non-degenerate dendrite $X$. Suppose that $e$ is an end point of $X$ such that $f_{i}(e)=e$ for all $i=1, \ldots, n$. Then there are $u, v \neq e \in X$ such that $f_{i}([e, v]) \cup f_{i}^{-1}([e, v]) \subset[e, u]$ for all $i=1, \ldots, n$.

COROLlary 2.5. Let $f$ be a homeomorphism on a non-degenerate dendrite X. Suppose that $e$ is an end point of $X$ such that $f(e)=e$. Then there is a sequence $\left\{u_{i}\right\}_{i=1}^{\infty}$ in $X$ satisfying the following two conditions simultaneously: (1) $\left[u_{1}, e\right] \supsetneq\left[u_{2}, e\right] \supsetneq\left[u_{3}, e\right] \supsetneq$ $\ldots$ and $\bigcap_{i=1}^{\infty}\left[u_{i}, e\right]=\{e\} ;(2)$ either $f\left(\left[u_{i}, e\right]\right) \subset\left[u_{i}, e\right]$ for all $i$ or $f^{-1}\left(\left[u_{i}, e\right]\right) \subset\left[u_{i}, e\right]$ for all $i$.

Let $X$ and $Y$ be metric spaces and let $f: X \rightarrow Y$ be continuous. If $\operatorname{diam}\left(f^{-1}(f(x))\right) \leq$ $\epsilon$ for some $\epsilon>0$ and for every $x \in X$, then $f$ is called an $\epsilon$-map. A continuum $X$ is tree-like provided that for every $\epsilon>0$ there is an $\epsilon$-map $f_{\epsilon}$ from $X$ onto some tree $Y_{\epsilon}$. 
THEOREM 2.6. [7, Exercise 10.50] A locally connected continuum is tree-like if and only if it is a dendrite.

THEOREM 2.7. [10, Theorem 1.1] Any action of a countable amenable group on a dendrite has a periodic point of order $\leq 2$.

\subsection{Rays and lines.}

Definition 2.8. Let $X$ be a compact metric space. If $\phi:[0,+\infty) \rightarrow X$ is a continuous injection, then $\phi$ or its image $R \equiv \phi([0,+\infty))$ is called a ray in $X ; R$ or $\phi$ is called oscillatory (respectively non-oscillatory) if $\bigcap_{n=0}^{\infty} \overline{\phi([n,+\infty))}$ contains at least two points (respectively only one point). If $\psi:(-\infty,+\infty) \rightarrow X$ is a continuous injection, then $\psi$ or its image $L \equiv \psi((-\infty,+\infty))$ is called a line in $X$; $L$ or $\psi$ is called oscillatory if either $\bigcap_{n=0}^{\infty} \overline{\psi((-\infty,-n])}$ or $\bigcap_{n=0}^{\infty} \overline{\psi([n,+\infty))}$ contains at least two points; is called bi-sided oscillatory if both $\bigcap_{n=0}^{\infty} \overline{\psi((-\infty,-n])}$ and $\bigcap_{n=0}^{\infty} \overline{\psi([n,+\infty))}$ contain at least two points; is called one-sided oscillatory if it is oscillatory but not bi-sided oscillatory; and is called non-oscillatory if it is not oscillatory.

We should note that if $\phi_{1}$ and $\phi_{2}$ are two rays with $\phi_{1}([0,+\infty))=\phi_{2}([0,+\infty))$, then $\phi_{1}$ and $\phi_{2}$ have the same types of oscillation. The same conclusion is true for lines. One may consult [3] for more information about rays (called 'quasi-arcs' in [3]).

Definition 2.9. Let $X$ be a compact metric space. Let $R$ be a ray in $X$ and let $L$ be a line in $X$. We say that $L$ is an extension of $R$ if there is a continuous injection $\phi:(-\infty,+\infty) \rightarrow X$ such that $L=\phi((-\infty,+\infty))$ and $R=\phi([0,+\infty))$.

The following lemma will be used in the proof of the main result.

LeMmA 2.10. Let $X$ be a uniquely arcwise connected continuum. Let $R$ be a ray in $X$ and let $\phi:[0,+\infty) \rightarrow X$ be a continuous injection such that $R=\phi([0,+\infty))$. If there is an arc $[a, b]$ in $X$ such that $\phi(0) \in(a, b)$, then $R$ can be extended to a line $L$ in X. Moreover, there is a maximal line extending $R$.

Proof. The first statement is clear. To show the second one, let

$$
\mathcal{F}=\{L: L \text { is a line which extends } R\} .
$$

It is clear that $\mathcal{F} \neq \emptyset . \mathcal{F}$ is a partially ordered set with respect to the inclusion of sets.

Assume that $\left\{L_{\lambda}\right\}_{\lambda \in \Lambda}$ is a totally ordered subset of $\mathcal{F}$. Set $N=\bigcup_{\lambda \in \Lambda} L_{\lambda}$. We claim that $N$ is a line which extends $R$.

In fact, for any $x \neq y \in N$, define $x<y$ if and only if exactly one of the following items holds: (1) $x, y \in R$ and $[\phi(0), x] \varsubsetneqq[\phi(0), y]$; (2) $x, y \notin R$ and $[x, \phi(0)] \supsetneq[y, \phi(0)]$; (3) $x \notin R$ and $y \in R$.

Then we can check that ' $<$ ' is a total ordering on $N$. By the compactness of $X$, for any integer $k>0$, there exists $a_{k} \in N \backslash R$ such that

$$
N \subset B\left(\left[a_{k}, \phi(0)\right], \frac{1}{k}\right) \cup R .
$$


Since $N$ contains no minimal element according to the ordering $<$, we may suppose that

$$
\cdots<a_{3}<a_{2}<a_{1}<0 .
$$

Let $\psi:(-\infty,+\infty) \rightarrow N$ be a continuous injection such that

$$
\psi([-n, 0])=\left[a_{n}, \phi(0)\right], \text { for all } n \in \mathbb{N}, \text { and } \psi([0,+\infty))=R
$$

It remains to show that $\psi((-\infty, 0))=N \backslash R$.

Assume on the contrary that there exists $z \in N \backslash \psi((-\infty,+\infty))$. Then $z<y$ for any $y \in \psi((-\infty,+\infty))$. Take $z^{\prime} \in N$ such that $z^{\prime}<z$ (noting that $N$ contains no minimal element). Then we have $d\left(z^{\prime},[z, \phi(0)]\right)>0$. This contradicts (2.1). Thus, the claim is proved.

Applying Zorn's lemma, we have proved the existence of the maximal line.

Example 2.11. In Example 1.1, $S^{-}$and $S^{+}$are one-sided-oscillatory rays; $S^{-} \cup S^{+}$is a bi-sided-oscillatory line; $L-(-1,1)$ is a non-oscillatory ray; $L-\{(-1,1),(-1,-1)\}$ is a non-oscillatory line; and the line $S^{-} \cup S^{+}$is an extension of the ray $S^{-}$.

2.4. Quasi-retractions. Let $X$ be a uniquely arcwise connected continuum. Let $Y$ be a tree, or an oscillatory ray, or a bi-sided-oscillatory line contained in $X$. Then, by the uniquely arcwise connectivity, for every $x \in X$, there is a unique $y \in Y$ such that $[x, y] \cap Y=\{y\}$; we denote $y=r_{Y}(x)$ and call the map $r_{Y}: X \rightarrow Y, x \mapsto r_{Y}(x)$ the quasi-retraction from $X$ onto $Y$. We should note that $r_{Y}$ is not continuous in general. The idea of quasi-retraction comes from [6].

Here we remark that the quasi-retraction $r_{Y}$ cannot be defined for any arcwise connected subset $Y$ of $X$ unless $Y$ satisfies the requirement that for every $x \in X$, there is a unique $y \in Y$ with $[x, y] \cap Y=\{y\}$; this is why we need to assume $Y$ being of some special form as above.

LEMMA 2.12. Let $X$ be a uniquely arcwise connected continuum. Let $Y$ be a tree, or an oscillatory ray, or a bi-sided-oscillatory line contained in $X$. If $Z$ is an arcwise connected subset of $Y$, then $r_{Y}^{-1}(Z)$ is an arcwise connected Borel measurable subset of $X$.

Proof. From the definition of $r_{Y}$, we see that if $x \in r_{Y}^{-1}(Z)$, then $\left[x, r_{Y}(x)\right] \subset r_{Y}^{-1}(Z)$, that is, every point in $r_{Y}^{-1}(Z)$ is connected to a point in $Z$ by an arc in $r_{Y}^{-1}(Z)$. Since $Z \subset r_{Y}^{-1}(Z)$ and $Z$ is arcwise connected, we know that $r_{Y}^{-1}(Z)$ is arcwise connected. For the measurability of $r_{Y}^{-1}(Z)$, one may consult [6].

Example 2.13. In Example 1.1, if $Y$ is the tree $L \cup B \cup R \cup M$, then $r_{Y}^{-1}((0,0))=S^{-} \cup$ $S^{+}$; if $Y$ is the line $S^{-} \cup S^{+}$, then $r_{Y}^{-1}((0,0))=L \cup B \cup R \cup M$; and if $Y$ is the ray $S^{-}$, then $r_{Y}^{-1}((0,0))=L \cup B \cup R \cup M \cup S^{+}$.

\section{Induced actions on dendrites}

In this section we will introduce the notions of the convex metrics and their completions; and the induced actions. 
3.1. Convex metrics and their completions. Let $X$ be a uniquely arcwise connected space (it need not be compact). A metric $d$ on $X$ is convex if, for any $u, v, x, y \in X$ with $[u, v] \subset[x, y]$, we have $d(u, v) \leq d(x, y)$. Suppose that $T_{1} \subsetneq T_{2} \subsetneq T_{3} \subsetneq \ldots$ is a strictly increasing sequence of trees contained in $X$. Let $T=\bigcup_{i=1}^{\infty} T_{i}$. Then $T$ is an arcwise connected subset of $X$. Clearly, $T$ is also the union of infinitely many arcs $I_{i}(i=1,2,3, \ldots)$ with $I_{i} \cap I_{j}$ being a point or empty for any $i \neq j$. Without loss of generality, we may suppose that

$$
T_{n}=\bigcup_{i=1}^{n} I_{i}
$$

for each $n \in \mathbb{N}$. Fix a homeomorphism $h_{i}: I_{i} \rightarrow[0,1]$ for each $i$. If $[a, b] \subset[0,1]$, we denote by $l([a, b])$ the length of the interval $[a, b]$ under the Euclidean metric on $[0,1]$, i.e. $l([a, b])=|a-b|$. For $x, y \in T$, define

$$
d(x, y)=\sum_{i=1}^{\infty} \frac{1}{2^{i}} l\left(h_{i}\left([x, y] \cap I_{i}\right)\right) .
$$

It is direct to check that $d$ is a convex metric on $T$. Let $\widetilde{T}$ be the completion of $T$ with respect to the metric $d$. We still use $d$ to denote the naturally induced metric on $\widetilde{T}$.

Proposition 3.1. $(\widetilde{T}, d)$ is a dendrite.

Proof. For every $\epsilon>0$, there is $n \in \mathbb{N}$ such that

$$
\sum_{i=n+1}^{\infty} \frac{1}{2^{i}}<\frac{\epsilon}{5} .
$$

Note that for every $\epsilon^{\prime}>0$, by the convexity of $d$, we always have

$$
d\left(r_{T_{n}}(x), r_{T_{n}}(y)\right) \leq d(x, y)<\epsilon^{\prime}
$$

whenever $x, y \in T$ with $d(x, y)<\epsilon^{\prime}$. This shows that $r_{T_{n}}:(T, d) \rightarrow\left(T_{n}, d\right)$ is uniformly continuous. So, $r_{T_{n}}$ can be extended to a continuous map

$$
r_{\epsilon}:(\widetilde{T}, d) \rightarrow\left(T_{n}, d\right) .
$$

We claim that $r_{\epsilon}$ is an $\epsilon$-map. Otherwise, there are $x, y \in \widetilde{T}$ with $d(x, y)>\epsilon$ and $r_{\epsilon}(x)=$ $r_{\epsilon}(y)$. Then, by the continuity of $r_{\epsilon}$ and the density of $T$ in $\widetilde{T}$, there are $x^{\prime}, y^{\prime} \in T$ such that $d\left(x, x^{\prime}\right)<\epsilon / 5, d\left(y, y^{\prime}\right)<\epsilon / 5$, and $d\left(r_{\epsilon}\left(x^{\prime}\right), r_{\epsilon}\left(y^{\prime}\right)\right)<\epsilon / 5$. So, by (3.2), we have

$$
d(x, y) \leq d\left(x, x^{\prime}\right)+d\left(x^{\prime}, r_{\epsilon}\left(x^{\prime}\right)\right)+d\left(r_{\epsilon}\left(x^{\prime}\right), r_{\epsilon}\left(y^{\prime}\right)\right)+d\left(r_{\epsilon}\left(y^{\prime}\right), y^{\prime}\right)+d\left(y, y^{\prime}\right)<\epsilon,
$$

which is a contradiction. By the arbitrariness of $\epsilon$, we get simultaneously that $(\widetilde{T}, d)$ is totally bounded and hence compact; is locally connected since $r_{\epsilon}$ is monotone by Lemma 2.12 (see [7, 8.4]); and is tree-like. It follows from Theorem 2.6 that $(\widetilde{T}, d)$ is a dendrite.

Remark 3.2. Though the inclusion $i:(T, d) \rightarrow X$ is a continuous injection, it is not necessarily an embedding. The topology on $(T, d)$ is the weak topology on $\left\{T_{n}\right\}$. That is, $A \subset T$ is $d$-closed if $A \cap T_{n}$ is closed for every $n$. 
Example 3.3. In Example 1.1, if we let $T_{n}=B \cup M \bigcup_{i=1}^{n}\left(I_{i} \cup I_{-i} \cup J_{i} \cup J_{-i}\right)$ and let $T=\bigcup_{n=1}^{\infty} T_{n}$, then the completion of $T$ with respect to the metric $d$ defined above is homeomorphic to the tree ' $\mathrm{H}$ '.

3.2. Induced actions. Let $X$ be a uniquely arcwise connected continuum. Let $G$ be a countable group acting on $X$. Suppose that $G=\left\{g_{i}: i=1,2,3, \ldots\right\}$. Take a point $p \in X$. For each positive integer $n$, let $S_{n}=\left\{g_{i}(p): i=1, \ldots, n\right\}$ and let $T_{n}=\left[S_{n}\right]$. Then we get an increasing sequence of trees:

$$
T_{1} \subset T_{2} \subset T_{3} \subset \cdots .
$$

Set $T=\bigcup_{n=1}^{\infty} T_{n}(=[G p])$. Then $T$ is a $G$-invariant uniquely arcwise connected subset of $X$. We assume that $T$ is not a tree. Then, by deleting some $T_{i}$ in (3.4) and renumbering the remaining $T_{i}$, we can assume that the sequence in (3.4) is strictly increasing. It follows from Proposition 3.1 that the completion $(\widetilde{T}, d)$ of $T$ with respect to the metric $d$ defined in (3.1) is a dendrite.

PROPOSITION 3.4. For each $g \in G$, the homeomorphism $g$ on $(T, d)$ is uniformly continuous with respect to the metric $d$.

Proof. Let $g \in G$. For every $\epsilon>0$, there is $m$ such that

$$
\sum_{i=m+1}^{\infty} \frac{1}{2^{i}}<\frac{\epsilon}{3} .
$$

Take a sufficiently large $n$ so that $S_{n} \supset S_{m} \cup g^{-1} S_{m}$. Then $g\left(S_{n}\right) \cap S_{n} \supset S_{m}$ and

$$
g\left(T_{n}\right) \cap T_{n} \supset T_{m} .
$$

By the compactness of $T_{n}$, there is $\delta>0$ such that

$$
d(g(x), g(y))<\frac{\epsilon}{3}
$$

whenever $x, y \in T_{n}$ with $d(x, y)<\delta$.

For any $u, v \in T$, let $u^{\prime}=r_{T_{n}}(u)$ and $v^{\prime}=r_{T_{n}}(v)$. Then, by the convexity of $d$, $d\left(u^{\prime}, v^{\prime}\right)<\delta$ whenever $d(u, v)<\delta$. Then, by (3.5)-(3.7), we have

$$
d(g(u), g(v)) \leq d\left(g(u), g\left(u^{\prime}\right)\right)+d\left(g\left(u^{\prime}\right), g\left(v^{\prime}\right)\right)+d\left(g\left(v^{\prime}\right), g(v)\right)<\epsilon
$$

provided that $d(u, v)<\delta$. This completes the proof.

From Proposition 3.4, we know that every $g \in G$ can be uniquely extended to a continuous map $\bar{g}:(\widetilde{T}, d) \rightarrow(\widetilde{T}, d)$. It follows that such an extension of $g^{-1}$ is an inverse to $\bar{g}$, whence we have the following proposition.

Proposition 3.5. For each $g \in G, \bar{g}:(\widetilde{T}, d) \rightarrow(\widetilde{T}, d)$ is a homeomorphism.

From Propositions 3.4 and 3.5 , we obtain an action of $G$ on the dendrite $(\widetilde{T}, d)$ by homeomorphisms, which is called the induced action from the $G$-action on $T$. 


\section{Proof of the main theorem}

In this section we start to prove the main result of the paper, namely Theorem 1.2. Let $X$ be a uniquely arcwise connected continuum and let $G$ be a countable amenable group. We want to show that every $G$-action on $X$ has a periodic point of order $\leq 2$.

Fix a point $p \in X$. Let $T=[G p]$ be the convex hull of its orbit. Then $T$ is an arcwise connected $G$-invariant subset of $X$. If $T$ is a tree, then $G$ has a periodic point of order $\leq 2$ in $T$ by Theorem 2.7. So, we may as well assume that $T$ is not a tree. Thus, by the discussion in $\S 3$, there is a metric $d$ on $T$ such that the completion $(\widetilde{T}, d)$ is a dendrite and there is an induced $G$-action on $(\widetilde{T}, d)$ by homeomorphisms. It follows from Theorem 2.7 that there is a periodic point $q \in \widetilde{T}$ of order $\leq 2$. If $q \in T$, then the conclusion of Theorem 1.2 holds, since $q \in X$.

So, we may assume that $q \in \widetilde{T}-T$, that is, $q$ is an end point of $\widetilde{T}$. If $q$ is a 2-periodic point of $G$, then $H \equiv\{g \in G: g(q)=q\}$ is a subgroup of $G$ with index two. Notice that $H$ is also amenable and $q$ is a fixed point of $H$. In this case, if we can show that $H$ has a fixed point $w \in X$, then $w$ is a periodic point of $G$ with order $\leq 2$ and the conclusion of Theorem 1.2 holds. So, to show Theorem 1.2 it remains to prove the following theorem.

THEOREM 4.1. If the induced $G$-action on $(\widetilde{T}, d)$ has a fixed point $q \in \widetilde{T}-T$, then $G$ has a fixed point in $X$.

Proof. We divide the proof into two steps.

Step 1: We assume first that $G$ is finitely generated with a generator set $\left\{g_{1}, \ldots, g_{n}\right\}$ for some $n \in \mathbb{N}$.

Fix a point $o \in T$; then $[o, q) \subset T \subset X$. Let $\phi:[0,+\infty) \rightarrow X$ be a continuous injection with $[o, q)=\phi([0,+\infty))$. Then $[o, q)$ becomes a ray.

Case 1. $\phi$ is non-oscillatory. Then there is $z \in X$ such that $z=\bigcap_{n=1}^{\infty} \overline{\phi([n,+\infty))}$. Since $q$ is fixed by every $g \in G$, we get from Corollary 2.5 a $g$ or $g^{-1}$-invariant decreasing sequence $\left[u_{i}, q\right]$ with $\bigcap_{i=1}^{\infty}\left[u_{i}, q\right]=\{q\}$. So, $\left[u_{i}, q\right)$ is a sequence of semi-open intervals in $X$, which is $g$ or $g^{-1}$ invariant. Thus, $z$ as a limit point of $u_{i}$ in $X$ is also $g$ invariant.

Case 2. $\phi$ is oscillatory. By Corollary 2.4, there are $c_{2}>c_{1}>0$ such that, for all $i=$ $1, \ldots, n$,

$$
g_{i}\left(\phi\left(\left[c_{2},+\infty\right)\right)\right) \cup g_{i}^{-1}\left(\phi\left(\left[c_{2},+\infty\right)\right)\right) \subset \phi\left(\left[c_{1},+\infty\right)\right) .
$$

Let $\prec$ be an ordering on $\phi([0,+\infty))$ defined by $\phi(t) \prec \phi(s)$ if and only if $t<s$ for any $t, s \in[0,+\infty)$.

First we assume that $z=\sup _{\prec}\left\{\left\{g\left(\phi\left(c_{2}\right)\right): g \in G\right\} \cap \phi\left(\left[c_{1},+\infty\right)\right)\right\} \prec+\infty$. We claim that $z$ is a fixed point of $G$. In fact, since $e \in G$, we have $\phi\left(c_{2}\right) \preceq z$. By the definition of $z$, we have $g_{i}^{-1}(z) \preceq z$ for each $1 \leq i \leq n$. As each $g_{i}$ preserves the ordering $\left.\prec\right|_{\phi\left(\left[c_{2},+\infty\right)\right)}$, the restriction of $\prec$ to $\phi\left(\left[c_{2},+\infty\right)\right)$, we have $z \preceq g_{i}(z)$, which implies that for each $1 \leq$ $i \leq n, g_{i}(z)=z$. That is, $z$ is a fixed point of $G$ and thus we get the conclusion. So, we may assume that

$$
\sup _{\prec}\left\{\left\{g\left(\phi\left(c_{2}\right)\right): g \in G\right\} \cap \phi\left(\left[c_{1},+\infty\right)\right)\right\}=+\infty .
$$


Consider the set $M=\bigcup_{g \in G} g\left(\phi\left(\left[c_{2},+\infty\right)\right)\right)$. Then $M$ is arcwise connected, since for any $h_{1} \neq h_{2} \in G$ there is some $c^{\prime}>0$ such that $h_{1}\left(\phi\left(\left[c_{2},+\infty\right)\right)\right) \cap h_{2}\left(\phi\left(\left[c_{2},+\infty\right)\right)\right) \supset$ $\phi\left(\left[c^{\prime},+\infty\right)\right)$ by Corollary 2.4. By Lemma 2.10 , we can take a maximal line $\psi$ : $(-\infty,+\infty) \rightarrow M \subset X$ with respect to the inclusion relation of subsets such that $\psi([0,+\infty))=\phi\left(\left[c_{2},+\infty\right)\right)$. Set $L=\psi((-\infty,+\infty))$.

Subcase 2.1. $\psi$ is bi-sided oscillatory in $X$. For each integer $n$, let $L_{n}=\psi((n, n+1])$ and let $K_{n}=\left\{x \in X: r_{L}(x) \in L_{n}\right\}$. By Lemma 2.12, each $K_{n}$ is an arcwise connected Borel measurable set in $X$. Clearly, these $K_{n}$ form a partition of $X$. Since $G$ is amenable, there is a $G$-invariant probability Borel measure $\mu$ on $X$. Suppose that $\mu\left(K_{m}\right)>0$ for some integer $m$. Since $\psi(m) \in M$, there is some $g^{\prime} \in G$ such that $g^{\prime}(\psi(m)) \in \phi\left(\left[c_{2},+\infty\right)\right)$, which implies that $r_{L}\left(g^{\prime}\left(K_{m}\right)\right) \subset \phi\left(\left(c_{2},+\infty\right)\right)$. Set $R=\phi\left(\left(c_{2},+\infty\right)\right)$. Then

$$
\mu\left(r_{L}^{-1}(R)\right) \geq \mu\left(g^{\prime}\left(K_{m}\right)\right)=\mu\left(K_{m}\right)>0 .
$$

However, by (4.2), we can take a sequence $s_{i} \in G$ such that $s_{1}\left(\phi\left(c_{2}\right)\right) \prec s_{2}\left(\phi\left(c_{2}\right)\right) \prec$ $s_{3}\left(\phi\left(c_{2}\right)\right) \prec \ldots \in R$ and $s_{i}\left(\phi\left(c_{2}\right)\right) \rightarrow+\infty$ as $i \rightarrow \infty$ with respect to the ordering $\prec$. Then we have

$$
0=\mu(\emptyset)=\mu\left(\bigcap_{i=1}^{\infty} s_{i}\left(r_{L}^{-1} R\right)\right)=\lim _{i \rightarrow \infty} \mu\left(s_{i}\left(r_{L}^{-1} R\right)\right)=\mu\left(r_{L}^{-1} R\right) .
$$

Since (4.3) and (4.4) contradict each other, this subcase does not occur.

Subcase 2.2. $\psi$ is one-sided oscillatory in $X$. Since $\phi$ is oscillatory, there must exist a point $z \in X$ such that $z=\bigcap_{n=1}^{\infty} \overline{\psi((-\infty,-n])}$. If $z$ is a fixed point of $G$, then the conclusion holds; otherwise, there is some $\tilde{g} \in G$ with $\tilde{g}(z) \neq z$. Since $\psi$ is maximal, there is $r \in(-\infty,+\infty)$ such that

$$
\psi([r,+\infty))=\psi((-\infty,+\infty)) \cap \tilde{g}(\psi((-\infty,+\infty))) .
$$

Denote $w=\psi(r) \in M$. Take $a \in(z, w)$ with $\tilde{g}(a) \in(\tilde{g}(z), w)$. Let $t \in(-\infty,+\infty)$ be such that $\psi(t)=a$. Set $L^{\prime}=L \cup\{z\}$ and set $P_{t}=\left\{x \in X: r_{L^{\prime}}(x) \in[z, a]\right\}$ and $Q_{t}=$ $\left\{x \in X: r_{L^{\prime}}(x) \in \psi((t,+\infty))\right\}$ (see Figure 2). Then, by Lemma 2.12, $P_{t}$ and $Q_{t}$ are arcwise connected and Borel measurable, and $X=P_{t} \cup Q_{t}$ (disjoint union). Since $G$ is amenable, there is a $G$-invariant Borel probability measure $\mu$ on $X$. Then $1=\mu(X)=$ $\mu\left(P_{t}\right)+\mu\left(Q_{t}\right)$. Noting that $\tilde{g}\left(P_{t}\right) \subset Q_{t}$, we have

$$
\mu\left(Q_{t}\right) \geq \mu\left(\tilde{g}\left(P_{t}\right)\right)=\mu\left(P_{t}\right)>0
$$

provided that $\mu\left(P_{t}\right)>0$. Thus, we always have $\mu\left(Q_{t}\right)>0$. Since $a \in M$, there is some $g \in G$ such that $g(a) \in \phi\left(\left[c_{2},+\infty\right)\right)$. Then, by an argument similar to that in Subcase 2.1, we get a contradiction.

Altogether, we finish the proof of Theorem 4.1 under the assumption that $G$ is finitely generated.

Step 2: Now suppose that $G$ is not finitely generated. For any finite subset $F$ of $G$, let $\langle F\rangle$ be the subgroup of $G$ which is generated by $F$. Define

$$
X_{F}=\{x \in X: x \text { is a fixed point of }\langle F\rangle\} .
$$




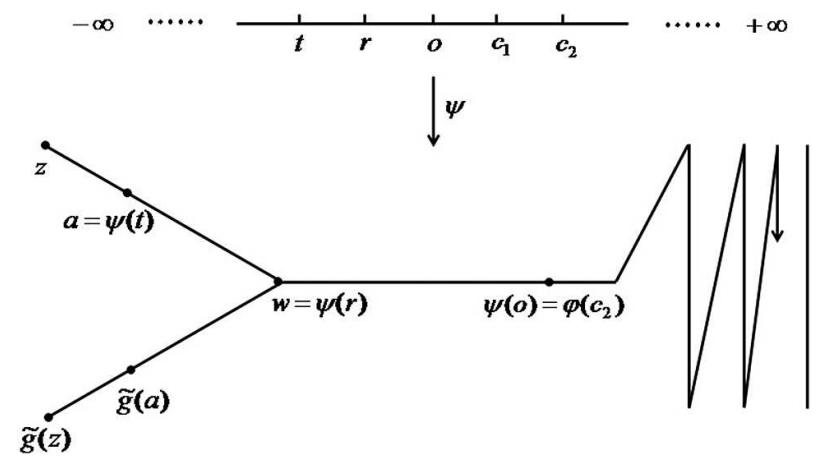

FIGURE 2. A maximal one-sided oscillatory line and its image.

Then $X_{F}$ is a non-empty closed subset of $X$. If $F^{\prime}$ is another finite subset of $G$, then $X_{F} \cap X_{F^{\prime}}=X_{F \cup F^{\prime}} \neq \emptyset$. Thus, the family of compact sets $\left\{X_{F}: F\right.$ is a finite subset of $\left.G\right\}$ has the finite intersection property. Hence,

$$
\bigcap\left\{X_{F}: F \text { is finite in } G\right\} \neq \emptyset,
$$

every point of which is a fixed point of $G$. Thus, we complete the proof of Theorem 4.1.

We end this paper with a remark. For any countable group $G$, if $p$ is a periodic point, then $[G p]$ is a tree. It follows that if for some bound $M$ every $G$-action on a tree has a periodic point with period at most $M$, then the same is true of any $G$-action on a uniquely arcwise continuum or else there exists an action of $G$ on such a space with no periodic points. Furthermore, our proof shows that if for some bound $M$ every $G$-action on a dendrite has a periodic point with period at most $M$, then the same is true of any $G$-action on a uniquely arcwise continuum which happens to admit an invariant measure.

Acknowledgements. We would like to thank Prof. Hanfeng Li for very helpful suggestions. We are also very thankful to the referee for valuable and thoughtful comments. The work is supported by NSFC (Nos. 11771318, 11790274, and 11431012).

\section{REFERENCES}

[1] R. H. Bing. The elusive fixed point property. Amer. Math. Monthly 76 (1969), 119-132.

[2] W. Holsztyński. Fixed points of arcwise connected spaces. Fund. Math. 69 (1969), 289-312.

[3] J. H. Mai and E. H. Shi. Structures of quasi-graphs and $\omega$-limit sets of quasi-graph maps. Trans. Amer. Math. Soc. 369(1) (2017), 139-165.

[4] R. Mańka. On uniquely arcwise connected curves. Colloq. Math. 51 (1987), 227-238.

[5] R. Mańka. On spirals and fixed point property. Fund. Math. 144 (1994), 1-9.

[6] L. Mohler. The fixed point property for homeomorphisms of 1-arcwise connected continua. Proc. Amer. Math. Soc. 52 (1975), 451-456.

[7] S. B. Nadler Jr. Continuum Theory. Marcel Dekker, Inc., New York, NY, 1992.

[8] A. L. T. Paterson. Amenability. American Mathematical Society, Providence, RI, 1988.

[9] E. H. Shi and B. Y. Sun. Fixed point properties of nilpotent group actions on 1-arcwise connected continua. Proc. Amer. Math. Soc. 137(2) (2009), 771-775. 
[10] E. H. Shi and X. D. Ye. Periodic points for amenable group actions on dendrites. Proc. Amer. Math. Soc. 145(1) (2017), 177-184.

[11] E. H. Shi and L. Z. Zhou. Periodic points of solvable group actions on 1-arcwise connected continua. Topology Appl. 157(7) (2010), 1163-1167.

[12] G. S. Young. Fixed-point theorems for arcwise connected continua. Proc. Amer. Math. Soc. 11 (1960), 880-884 